\title{
Study on Strategies for Coping With Public Health Emergencies in Rural Areas--Based on the Case Analysis of S Town, Sichuan
}

\author{
Guihua Liu ${ }^{1,}$ Yu Feng ${ }^{2, *}$ Pu Zhang ${ }^{3}$ \\ ${ }^{1-3}$ School of Public Affairs and Law, Southwest Jiaotong University, Chengdu, Sichuan 610031, China \\ ${ }^{*}$ Corresponding author. Email: $894033641 @$ qq.com
}

\begin{abstract}
The sudden outbreak of COVID-19 has not only seriously threatened the safety of people's life and property, but also brought severe challenges to governments at all levels. Due to the relative lack of information and medical resources in rural areas, the problem of ineffective response to epidemic situation is inevitable. This paper analyzes the prevention and control measures of S town in Sichuan Province. On this basis, it further investigates the problems in the response to public health emergencies in rural areas, and puts forward corresponding improvement measures so as to provide reference for China's public health emergency management system.
\end{abstract}

Keywords: COVID-19, public health emergencies, rural areas, emergency management

\section{INTRODUCTION}

Public health emergency refers to the major infectious diseases, mass diseases of unknown causes, major food and occupational poisoning accidents and other events that break out and probably do great harm to public health. This definition refers specifically to major public health events and has a narrow meaning. The broad meaning further includes general public health events that occur suddenly and cause general damage to public health [1]. Public health emergencies have the characteristics of suddenness, unconventionality and danger [2], which may bring about serious harm in a certain area or even the entire country. Therefore, the effective emergency response and disposal of public health emergencies is an important guarantee for national health, which is also an important test for a country or a government's governance capability. The new coronavirus pneumonia epidemic is a major public health emergency that has occurred in China since founded with the fastest spread, the widest infection range, and the most difficult prevention and control. This outbreak, from Wuhan to wider districts in China and then even to the whole world, has caused a seriously adverse impact on the normal life order of the society, economic development, and the safety of the people's lives. The risk of rural epidemic outbreaks cannot be ignored either. Due to the special occasion of the Spring Festival, the "spring travel rush" has brought serious challenges to the prevention and control of epidemic outbreaks in rural areas. Therefore, correctly guiding rural areas to carry out epidemic prevention and control, sorting out the problems in rural epidemic prevention and control, as well as exploring countermeasures for that are of great significance in the aspect of improving the emergency management of rural public health emergencies. This article, taking S Town, Sichuan Province as an example, will conduct field research on the issues mentioned before based on current prevention and control measures.

\section{CASE}

This paper adopts a case study method to reflect the problems of emergency management and response at the community level in China and then to put forward relevant measures, by presenting the emergency management situations and the process of response in the $\mathrm{S}$ town during the outbreak. The Covid-19 crisis is a major test for the government's governance capacity. Moreover, rural areas have become the key regions that necessitate strengthening epidemic prevention and control due to their weak health systems and large population of outsiders. The continuous spread of the epidemic indeed turns into a severe test for the ability of governance and emergency prevention of rural officials. In order to win the battle with Covid-19, the city and the whole province where $\mathrm{S}$ town is located have made a quick response in the early stage of the outbreak. A large number of policies have been intensively issued in response to the issues in rural areas. These measures have been continuously refined, and all parties from various fields have adopted different measures: the $S$ town government set up an emergency command team at the first time and urged each department to perform their respective duties, to strengthen the coordination among different departments, and to ensure that everything is carried out steadily and orderly; the local health center timely designated the epidemic area and drew a line, strengthening the investigation of people who returned from areas with high incidence of epidemics, then carrying out effective prevention and control; the village 
(residential) committee is the specific implementer of relevant policies, and its main responsibility is to ensure that the prevention and control measures in certain area are truly followed and carried out, as well as to take advantage of broadcasts, mobile publicity vehicles, paper flyers and banners to inform residents about the basic information of COVID-19, the latest epidemic situations and some prevention tips and so on. These are all what has been done in this region in its process of prevention and control.

\section{PROBLEMS IN RURAL AREAS}

In this case, $\mathrm{S}$ town rapidly takes relevant measures after COVID-19 outbreak and to some extent has achieved relatively satisfying effect in the aspect of preventing further expansion of epidemic. However, there are still some defects in terms of the whole process of epidemic prevention and control in $\mathrm{S}$ town rural areas, which also exposed the problems of public health emergency response in overall rural areas. These problems are as follows:

\subsection{Backward Medical Conditions and Insufficient Medical Resources}

In the emergency management of public health emergency, the hospital plays an extremely important role as their specific conception and awareness, their work plan and the allocation of resources will all affect the implementation of emergency management [3]. However, the unbalanced development of urban and rural areas under the influence of their dual structures finally leads to the unbalanced development of urban-rural health systems. Currently, emergency medical workers are mainly in central urban areas rather than rural areas, which leads to the imbalance of medical personnel in rural areas. Therefore, it is difficult for rural districts to deal with complex and changeable events only depending on the limited existing emergency personnel, which greatly lowers the efficiency of rural emergency management. Secondly, inadequate health resources and poor public health conditions prevent rural areas from obtaining services of the same quality as urban areas, which is probable to give rise to inadequate efforts of prevention and control.

\subsection{Lower Awareness of Health Risks for Rural Residents}

The residents are not only the main participants, but also the main objects of risk management when a public health emergency occurs. The residents' prevention awareness of health risks is very important for the disposal of public health emergency. At present, rural residents are not welleducated and lacking in public health knowledge. Most people have not paid enough attention to their own health risks and the whole situations of public health. Their incorrect conceptions have brought some hidden dangers for the spread of the epidemic and also made it more difficult for community-level governments to prevent and control the epidemic. In the early stage of the COVID-19 outbreak, many people considered that the outbreak would not occur in rural areas. Thus, some rural residents let their guard down and still refused to wear masks when going outside to attend family gathering and dine with friends. Although the health safety work in China has achieved great success since the reform and opening up, the basic understanding and the sense of responsibility of health for rural residents still need to be improved due to the relatively closed geographical environment. Furthermore, this contradiction is particularly prominent when dealing with the outbreak of some major epidemic.

\subsection{Insufficient Emergency Management Capabilities for Some Community-Level Leaders}

The effectiveness of emergency management in rural areas depends to the ability of community-level leading officials. Generally, the government will take centralized measures to prevent and control the emergency as a result of its special nature so as to centralize the power and resources. On the one hand, due to the suddenness of public health emergency, it's quite different compared to the previous routine management. under the circumstances like limited information and resources, and even no similar precedent to learn, managers are required to make scientific analysis and assessment of the epidemic situations and make correct decisions. On the other hand, because of the rapid spread and the changeable characteristics and great harmfulness of the epidemic outbreak, we must consider the timeliness of the decision. Even a perfect plan may still miss the best time for response if hesitating and mulling over repeatedly. However, most of the rural community officials, lack of relevant knowledge, are unable to make scientific analysis and decisions when dealing with public health emergency, which brings about the failure of the first defense line in public health emergency management.

\subsection{Insufficient Emergency Material Reserves and Limited Emergency Funding}

In terms of emergency supplies, it is difficult for rural areas to cope with sudden public health emergency. In the early stage of the epidemic, all districts of China were caught in the predicament of extremely scarce supplies. Necessary anti-epidemic materials such as masks and disinfectants on the market are in short supply, and more seriously some sellers even raise the price for several times, which is as a result of the Spring Festival holiday when most factories suspend production. This problem particularly stands out in rural areas. As for emergency funding, the capital investment determines whether the 
emergency management mechanism can be more effectively established and improved. The capital investment of this part is controlled by the government finance. For some emergency funds needed in the areas of monitoring and early warning, material reserves and the rehearsal training for community departments, it is difficult to establish an effective and sustainable input guarantee mechanism for a long term [4]. Therefore, funding is still a key bottleneck in the emergency management mechanism for public health in rural areas.

\section{STRATEGY PATH DIAGRAM}

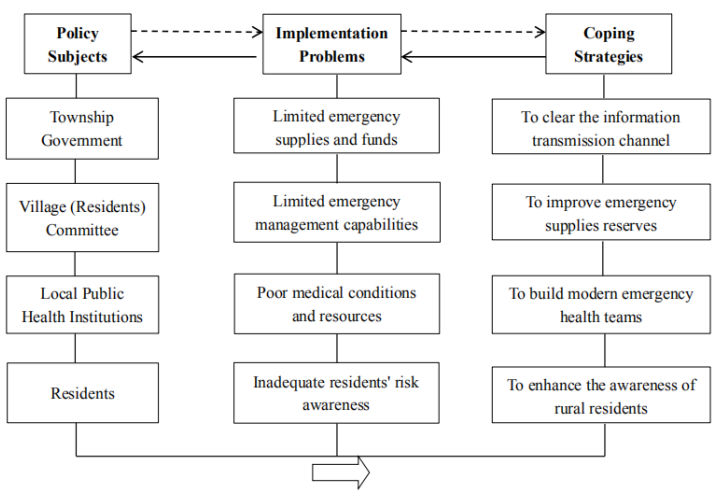

Figure 1 Strategy path transmission in the case

From case analysis, we can conclude the problems in the response of public health emergency in rural areas as limited emergency supplies and funds, low risk consciousness for residents, inadequate capability of emergency management, insufficient medical conditions and resources. In order to solve those problems, all parties including the government, the village committee, the public health institutions at the community level and the residents should take effective measures. Therefore, it's necessary to form relevant measures in a problem-oriented perspective, and the successful solution of those problems will in turn help all subjects to develop experience, which finally makes up a complete policy path.

\section{STRATEGY PATH ANALYSIS}

Through the case of town $\mathrm{S}$, it can be found that in the strategy path transmission shown in Figure 1, the main subjects involved in the policy include township government, village committee, public health institutions, and residents. With the participation of these subjects, some problems in the actual implementation of rural areas occurred in the early and mid-term public health emergencies. Later work should be promoted and improved through the following strategies.

\subsection{Opening up Information Transmission Channel by Using Networked Means}

In order to effectively prevent the spread of infectious diseases in a short period of time, it is necessary to be able to obtain information on related events in a timely manner and respond quickly to this. Therefore, a scientific and effective emergency management information network platform must be established and perfected. The accurate analysis of relevant data and information can provide favorable support for the command system to carry out relevant countermeasures and actions [5]. Local government should pay attention to the use and update of information systems. Through the information construction, they can integrate and use relevant information. At the same time, the health emergency network can be exte nded to towns and villages and various public health institutions. The township government can achieve full coverage of rural emergency information by establishing an emergency SMS platform. It can not only inform residents of the basic situation of the epidemic situation, but also spread scientific and effective prevention and control knowledge. It can also break down some rumors during the epidemic in time. In addition, the grass-roots government can use public opinion to guide the residents, so as to soothe their emotions and avoid the behaviors that affect public order caused by negative mentality. At the same time, it is also necessary to ensure the transparency of information. Relevant departments should release relevant authoritative information in a timely manner, and should also spread scientific rescue methods to the society. In this way, the public is prevented from concealing the illness because of their fear, so as not to cause greater harm to society.

\subsection{Promoting the Construction of Emergency Health Care Teams}

The public health human resources in rural areas are the front-line team in primary health care in China, and they bear the most basic health security, prevention and medical burdens of residents in rural areas. First, we should strengthen the technical capabilities of medical staff at the grassroots level and carry out emergency management education and training. In this way, medical staff can better carry out disease prevention and monitoring, organize and form epidemic reports, and improve emergency measures. And in this process, it is able to respond maturely in the face of emergencies. Second, the training of emergency health personnel in rural areas should aim at cultivating compound talents, so as to strengthen the reserve of grassroots public health personnel. The local government must try to increase the proportion of its senior health management talents and formulate a reasonable talent introduction plan. It is necessary to introduce the most urgently needed and suitable professionals, as well as provide appropriate 
treatment to attract and retain talents, so as to establish a reasonable talent echelon.

\subsection{Enhancing Rural Residents' Emergency Awareness}

At present, rural residents still lack risk awareness and emergency response capabilities. Therefore, on the one hand, the public health management department should actively strengthen the health and safety education of the grassroots and improve their public health awareness; at the same time, the rural authorities should use feasible means to implement publicity and education under the role of public platforms. We must be good at finding out the blind spots of the grassroots, and adopt different methods to carry out education for different levels. In the process of hierarchical education, it is necessary to make residents deeply understand the management of public health emergencies. And the rural areas should be strongly supported to improve the management level of public health emergencies in the participation of the whole people. On the other hand, people should be encouraged to exert their enthusiasm. With regular drills, the public can enhance their emergency awareness. It is also possible to implement knowledge competitions and simulation drill competitions through the Internet, media and lectures, so that residents can truly transform their knowledge of responding to public health emergencies into practical capabilities.

\subsection{Improving Emergency Supplies Reserves}

For the uncertainties of public health emergencies, adequate reserves of emergency resources are the key to a smooth solution. Therefore, at the beginning of an emergency, effective measures must be taken quickly to achieve a reasonable allocation of resources. On the one hand, detection and disposal configuration should be increased. According to the requirements of the Ministry of Health, corresponding emergency equipment should be deployed at the grassroots level. In rural areas, infrastructure construction should be improved and implemented, and some advanced medical equipment, especially disease surveillance equipment, should be purchased in the form of financial subsidies. In addition, relevant material reserves should be increased, such as medical equipment, medical treatment, etc. Ensure that resources can be used reasonably to avoid waste or idleness of resources. On the other hand, it is necessary to increase the special fund input of the local government. Special fund input is the fundamental guarantee for the relevant material reserves in case of emergency. This investment should not only be prepared at the critical moment, but also set up under normal conditions to prepare for unexpected needs. In addition, we must focus on the problem that the categories of emergency material reserve were too single. It is also necessary to achieve both capital reserve and material reserve. And Attention should be paid to the construction of insurable infrastructure and a unified plan [6].

\section{CONCLUSION}

This study starts from a specific case and discusses the emergency management of rural areas in China in response to public health emergencies. We found that the sensitivity of the epidemic situation in rural areas and public awareness need to be improved at this stage. In short, localities should consider their own actual situation and improve the emergency response capacity in rural areas from the aspects of personnel training and public education. It is of great significance to take precautionary measures to protect people's health and maintain social stability.

\section{REFERENCES}

[1] EnzhiWu, Zhuliang Yu, Discussion on the problems existing in response to emergencies in grassroots public health departments, Zhejiang Preventive Medicine, 2005(10): 65-74. DOI: https:// 10.19485/j.cnki.issn10070931.2005.10.047

[2] Wei Na, Research on emergency Management of Public health Emergencies facing grassroots government, Northeastern University, 2008.

[3] Yan Qiu, Analysis of emergency management of public health emergencies in hospitals and countermeasures, Journal of general stomatology, 2019, 6(36): 12. DOI: https:// 10.16269/j.cnki.cn119337/r.2019.36.009

[4] Dongyan Lu, Current situation and countermeasures of emergency management of public health emergencies in China, Journal of community medicine, 2016, 14(24): 6569.

[5] Haitao Wang, Reflections on emergency Response of grassroots Communities in Public health Emergencies, Democracy and Law Times, 2020-02-22(003). DOI: https:// 10.28579/n.cnki.nmzfz.2020.000281

[6] Jie Xiong, Jialiang Dong, Federation of Industry and Commerce of Jiangsu Province, Ten Countermeasures to Strengthen the construction of public health emergency support, China Industrial and Commercial Times, 202002-27(003) DOI:

10.38311/n.cnki.nzhgs.2020.000702 